\title{
(2) OPEN ACCESS \\ COVID-19-related healthcare closures negatively affect patient health and postoperative recovery
}

\author{
Michaela Kopka, ${ }^{1}$ Julie-Anne Fritz, ${ }^{1}$ Laurie Anne Hiemstra (1) , ${ }^{1,2}$ Sarah Kerslake (1) ${ }^{1}$
}

'Banff Sport Medicine Clinic \& Foundation, Banff, Alberta, Canada

2Department of Surgery, University of Calgary, Calgary, Alberta, Canada

\section{Correspondence to} Dr Michaela Kopka, Banff Sport Medicine Clinic, Banff, T1L 1B3, Canada;

kopka@banffsportmed.ca

Accepted 1 August 2020 Published Online First 6 September 2020
Check for updates

(C) International Society of Arthroscopy, Knee Surgery and Orthopaedic Sports Medicine 2020. Re-use permitted under CC BY-NC. No commercial reuse. Published by BMJ.
ABSTRACT
Objective To determine how COVID-19-related healthcare closures affect the health, recovery and access to resources of preoperative and postoperative orthopaedic sport medicine patients.

Methods Patients whose orthopaedic restorative surgeries were postponed as well as those within 3 months postoperative completed electronic questionnaires assessing physical health, emotional health, virtual care and access to resources. The EuroQoLfive-dimensional-three level (EQ-5D-3L) was included as a quantitative and standardised measure of general health status. Data were descriptively analysed using means, SD and qualitative measures.

Results A total of 115 patients whose surgeries were postponed completed the survey. Notable findings included that $41.7 \%$ of patients reported an increase in their symptoms during the COVID-19-related closures, and $68.7 \%$ described a negative impact on their physical health. Furthermore, $62.6 \%$ of patients reported that the postponement of their surgery would negatively affect their ability to return to work. A substantial percentage of patients $(71.3 \%)$ reported that postponement of their surgery had an overall negative impact on their physical and/or emotional health. The mean health state on the EQ-5D-3L visual analogue scale (VAS) scale was 69.9 (SD=18.7). A total of 198 patients within 3 months postoperative completed the survey. A majority of patients (69.7\%) indicated that COVID-19-related healthcare closures had an overall negative affect on their recovery. One-third of patients (35.4\%) stated that they experienced increased symptoms as a direct result of limited postoperative follow-up. Less than half $(41.4 \%)$ of patients accessed virtual physiotherapy, and only $42.9 \%$ of those that did found it to be helpful in some way. Virtual orthopaedic follow-up was deemed helpful by $61.3 \%$ of patients but many commented that it did not replace inperson visits. The mean health state reported on the EQ-5D-3L VAS scale was 76.9 ( $S D=15.6)$.

Conclusion This study highlighted the significant impact of COVID-19-related healthcare closures on preoperative and postoperative orthopaedic sport medicine patients. It revealed that restorative procedures play an important role in maintaining physical and emotional health, as well as returning individuals to work and leisure activities. It also showed that virtual care did not adequately replace the hands-on assessments of allied care and orthopaedic specialists.

Level of Evidence $V$.

\section{INTRODUCTION}

The COVID-19 outbreak was first described in Wuhan, Hubei, China in December 2019. On 11

\section{What are the new findings}

- $41.7 \%$ of patients whose restorative surgeries were postponed due to COVID-19 experienced increased symptoms related to their injury.

- $62.6 \%$ of postponed patients described a negative effect on their ability to return to work.

- $71.3 \%$ of early postoperative patients reported a negative impact on their recovery.

- Limited access to hands-on physiotherapy was the most commonly reported barrier to postoperative recovery.

March 2020, WHO declared a global pandemic. In response, nations around the world enacted strict travel restrictions, self-isolation requirements and closures of non-essential services. To decrease the burden on the healthcare system, many countries postponed non-urgent medical procedures including elective surgeries. ${ }^{1}$ In Canada, all restorative orthopaedic surgeries as well as supportive healthcare services such as physiotherapy were shut down in mid-March.

As lockdown measures are lifted and healthcare services resume, many questions remain surrounding the allocation of scarce resources and restarting of non-urgent procedures. With the necessary safety measures in place to protect healthcare workers and patients from COVID-19 infection as well as the possibility of a second wave, the medical system is likely to function at a reduced capacity for some time. ${ }^{23}$ Accordingly, information on the impact of the closure is needed in order to formulate an appropriate and equitable reopening strategy.

In an effort to understand the impact of COVID19-related healthcare closures on orthopaedic sport medicine patients in our practice, a questionnaire was distributed to all preoperative and early postoperative patients. The survey was electronically administered to two groups of patients: those whose surgeries were postponed due to the operating room closures and those in their first 3 months postoperative when the COVID-19-related closures came into effect. A series of questions pertaining to physical and emotional health, virtual care and access to resources were posed. The goal of this study was to determine how COVID-19-related healthcare closures affect patient health, recovery, and access to resources.

\section{METHODS}

Patients whose restorative surgeries were postponed and those within 3 months of surgery were 
electronically sent distinct surveys on 1 May 2020. The surveys were designed as a quality assurance assessment and responses were anonymous. All patients who responded to the questionnaires indicated their consent to provide information at the beginning of the survey.

The survey questions were organised into four key themes: physical health, emotional health, virtual care and access to resources. Responses employed a 7-point Likert scale. The EuroQoL-five-dimensional-three level (EQ-5D-3L) was included as a quantitative and standardised measure of general health status. Patients received reminder emails at 1 week and again at 2 weeks after survey distribution. The surveys were closed 4 weeks following distribution.

Data were descriptively analysed using means, SD and qualitative measures. Due to the nature of this research, further formal statistical analysis was not performed.

\section{RESULTS}

\section{Postponed patients}

Of the 269 patients whose restorative surgeries were postponed as a result of COVID-19, 147 returned the survey. Eleven were excluded due to incomplete responses and 21 were excluded because they underwent surgery prior to completing the questionnaire. In total, 115 patients were included in the analysis. The most frequently postponed procedures were anterior cruciate ligament (ACL) reconstruction, shoulder surgery (primarily arthroscopic stabilisation or rotator cuff repair) and meniscal surgery. Other procedures included: patellar stabilisation, multiligament knee reconstruction and high tibial osteotomy. The majority of patients were between 18 and 55 years of age $(78.3 \%) ; 5.2 \%$ were $<18$ years of age and $16.5 \%$ were $>55$ years of age.

Three-quarters $(75.7 \%)$ of patients stated that they experienced pain as a result of their injury, and $51.1 \%$ indicated that their pain increased during the COVID-19-related closures. A small percentage of patients (7.4\%) reported that their pain decreased due to absence from work and exacerbating activities; $41.5 \%$ experienced no change (figure 1). Overall, $41.7 \%$ reported that their symptoms increased during the COVID19-related closures, and $68.7 \%$ described a negative impact on their physical health. Notably, $62.6 \%$ of patients reported that the postponement of their surgery would negatively affect their ability to return to work (figure 2). In their comments, patients stated that their injury was preventing them from working in their field and postponement of surgery would further delay their ability to return to full function. Others noted that the

\section{Percent (\%) of patients reporting a change in pain levels during COVID-19 shutdowns}

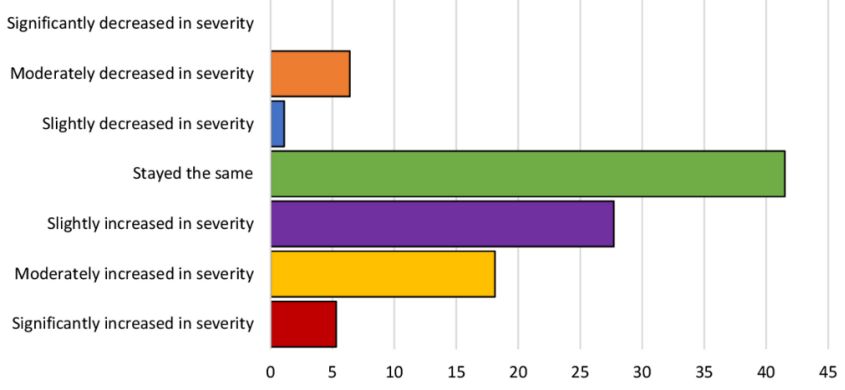

Figure 1 Patients were asked to report how the level of pain they were experiencing as a result of their injury had changed during the COVID-19related shutdown
Precent (\%) of patients reporting affects on their ability to return to work due to the postponement of their surgery

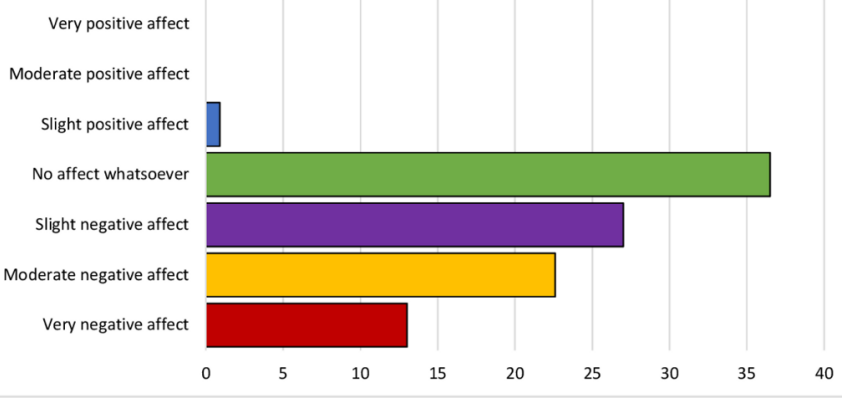

Figure 2 Patients were asked to what extent the postponement of their surgery had affected (or will affect) their ability to return to work.

financial burden of COVID-19 would impact their ability to take time off work for postoperative recovery. The majority of patients $(90.4 \%)$ indicated that postponing their surgery would also negatively affect their ability to return to sport and leisure activities.

Nearly three-quarters of patients $(71.3 \%)$ indicated that postponement of their surgery had an overall negative impact on their physical and/or emotional health. The majority (79.1\%) expressed negative emotions as a result. Common feelings described included: anxiety/stress (26.5\%), frustration $(42.6 \%)$ and concern $(20.6 \%)$. Less than half $(39.1 \%)$ of patients were able to access resources for support. The most common resources accessed included physiotherapy $(21.7 \%)$, orthopaedic surgeon $(7.8 \%)$ and online content $(8.7 \%)$.

With respect to the EQ-5D-3L, patients reported difficulties with mobility (49.6\%) and performing their usual activities (66.1\%). A large majority experienced pain and discomfort $(82.6 \%)$ and $46.1 \%$ noted anxiety and depression as a result of the COVID-19 closures (table 1). The mean health state reported on the visual analogue scale (VAS) scale (0-100) was $69.9(\mathrm{SD}=18.7)$.

\section{Postoperative patients}

Of 476 patients who underwent restorative surgeries in the 3 months prior to the COVID-19-related closures, 208 returned the survey. Ten patients were excluded due to incomplete responses. In total, 198 patients were included in the analysis. The most common surgical procedures reported were ACL reconstruction, patellar stabilisation and meniscal surgery. Other procedures included: arthroscopic shoulder stabilisation, rotator cuff repair, multiligament knee reconstruction and tibial tubercle osteotomy. The majority of patients were between 18 and 55 years of age $(87.8 \%) ; 4.6 \%$ were $<18$ years of age and $7.6 \%$ were $>55$ years of age.

Nearly half of all patients (43.4\%) reported that they were behind in their postoperative recovery, and $69.7 \%$ felt that COVID-19-related healthcare closures had an overall negative affect on their recovery (figure 3). One-third (35.4\%) stated that they experienced increased pain or other symptoms as a direct result of limited postoperative follow-up. More than half of patients (60.1\%) expressed at least one negative emotion related to the COVID-19 closures. Common feelings described included: concern (41.4\%), stress/anxiety (40.9\%) and frustration $(20.7 \%)$. Some patients $(14.7 \%)$ indicated that they felt motivated and $17.7 \%$ described some positive emotions.

Less than half $(41.4 \%)$ of patients accessed virtual physiotherapy, and only $42.9 \%$ of those did reported that it was helpful 
Table 1 EQ-5D-3L frequencies reported by problem and level for patients that had surgery postponed

\begin{tabular}{|c|c|c|c|c|c|}
\hline & $\begin{array}{l}\text { Mobility } \\
\mathrm{n}(\%)\end{array}$ & $\begin{array}{l}\text { Self-care } \\
\mathrm{n}(\%)\end{array}$ & $\begin{array}{l}\text { Usual activities } \\
\mathrm{n}(\%)\end{array}$ & $\begin{array}{l}\text { Pain/Discomfort } \\
\mathrm{n}(\%)\end{array}$ & $\begin{array}{l}\text { Anxiety/Depression } \\
\mathrm{n}(\%)\end{array}$ \\
\hline No problems & $58(50.4)$ & $106(92.2)$ & $39(33.9)$ & $20(17.4)$ & $62(53.9)$ \\
\hline Any problems & 57 (49.6) & $9(7.8)$ & $76(66.1)$ & 95 (82.6) & $53(46.1)$ \\
\hline Total & $115(100)$ & $115(100)$ & $115(100)$ & $115(100)$ & $115(100)$ \\
\hline
\end{tabular}

EQ-5D-3L, EuroQoL-five-dimensional-three level.

in some way. Younger patients were more likely to use virtual healthcare resources than older individuals, with $55.9 \%$ in the 18-24 years age group reporting that they had accessed virtual physiotherapy compared with only $27.3 \%$ in the $>45$ years age group (online supplementary table 1 ). The majority of patients (73.2\%) felt that lack of hands-on physiotherapy had negatively impacted their recovery (figure 4). Limited access to physiotherapy was the most commonly reported barrier to recovery (64.1\%). Other barriers included lack of time, inadequate guidance with postoperative exercises and lack of access to exercise equipment. With regard to virtual orthopaedic follow-up, $61.3 \%$ indicated that they found it helpful, but many felt that it did not replace in-person care. Some comments included:

Hearing only what is needed to be done is less effective than seeing, hearing, and doing.

Definitely answered questions but can't replace in person visits.

Only $37.9 \%$ of the respondents indicated that lack of inperson orthopaedic follow-up had negatively impacted their recovery, while $59.6 \%$ were neutral.

The majority $(86.9 \%)$ of patients were able to follow the postoperative rehabilitation protocols provided by the surgical office, and $93 \%$ were able to perform some exercises at home. Other resources that patients accessed during the COVID-19-related closures included: physiotherapy (61.1\%), surgeon's office $(30.3 \%)$ and online educational resources (28.8\%). Fifteen per cent indicated that they did not access any additional resources.

With respect to the EQ-5D-3L, patients reported symptoms of pain/discomfort $(77.3 \%)$ as well as difficulties with performing their usual activities (56.1\%). One-third (34.8\%) noted anxiety and depression as a result of the COVID-19 closures, and 28.3\% expressed mobility restrictions (table 2 ). The mean health state reported on the VAS scale (0-100) was $76.9(\mathrm{SD}=15.6)$.

\section{DISCUSSION}

This is the first study to investigate the impact of COVID-19related healthcare closures on orthopaedic sport medicine

Percent (\%) of patients reporting how COVID-19 shutdowns are perceived to be affecting their recovery

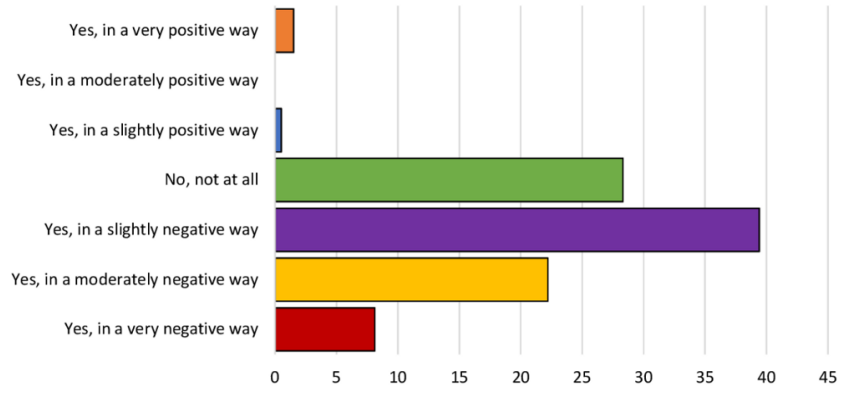

Figure 3 Patients were asked if they were worried that the COVID-19related shutdowns had affected their postoperative recovery. patients. Patients whose restorative surgeries were postponed, as well as those in the early postoperative period ( $<3$ months) were asked a series of questions pertaining to their physical and emotional health, barriers to recovery and access to resources. The findings of this study revealed that, in both groups, the COVID-19-related closures had an overall negative impact on physical and emotional health and resulted in significant barriers for accessing necessary care. Specific to the EQ-5D-3L, both the postponed and early postoperative patient groups demonstrated substantially lower scores in the domains of mobility, usual activities, pain/discomfort and anxiety/depression, compared with population norms. ${ }^{4}$ The mean VAS health state score for the postponed group ranged 11-30 points lower than age-matched populations. $^{4}$

Patients whose surgical procedures were postponed due to COVID-19 indicated that they experienced physical pain due to their injury, and 51.1\% stated that their symptoms increased as a result of the healthcare closures. In a study by Endstrasser et al, ${ }^{5}$ telephone interviews were conducted with 63 patients awaiting hip and knee arthroplasty at the beginning, middle and end of the COVID-19 lockdown. The authors found a significant increase in VAS pain scores, a decrease in SF-12 physical component scores and a decrease in Tegner activity scores over the course of the lockdown. Although not unexpected in a population of patients suffering with end-stage hip and knee osteoarthritis, the increase in physical pain and symptoms reported by a typically healthy and high-functioning cohort of sport medicine patients is concerning. Another important finding of this study was that $62.6 \%$ of postponed patients reported that the surgery delay would have a negative effect on their ability to return to work. This finding underscores the impact of restorative surgeries on physical function, quality of life and the overall societal burden of disease.

A significant number of patients (69.7\%) who were in the initial 3-month postoperative period described a negative impact on their recovery as a result of the COVID-19-related healthcare closures. Inability to access hands-on physiotherapy was

Percent (\%) of respondents reporting how they perceive the lack of in-person physiotherapy has impacted their recovery

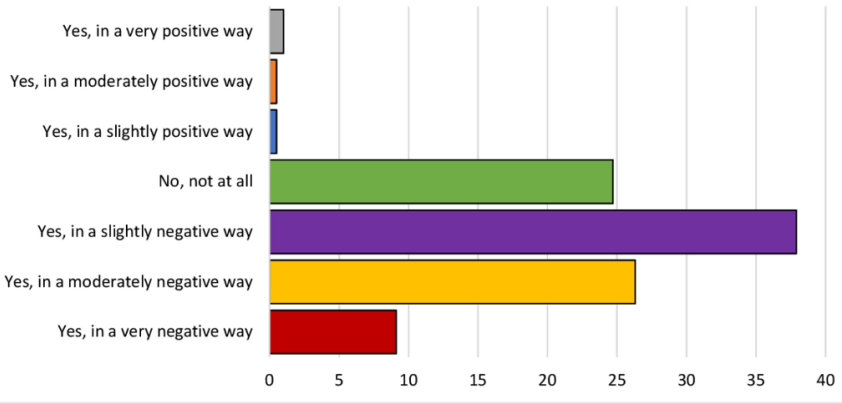

Figure 4 Patients were asked to report how they felt the lack of inperson access to physiotherapy had impacted their postoperative recovery. 
Table 2 EQ-5D-3L frequencies reported by problem and level for patients that were up to 3 months postoperative

\begin{tabular}{llllll} 
& $\begin{array}{l}\text { Mobility } \\
\mathbf{n}(\%)\end{array}$ & $\begin{array}{l}\text { Self-care } \\
\mathbf{n}(\%)\end{array}$ & $\begin{array}{l}\text { Usual activities } \\
\mathbf{n}(\%)\end{array}$ & $\begin{array}{l}\text { Pain/Discomfort } \\
\mathbf{n}(\%)\end{array}$ & \multicolumn{2}{l}{$\begin{array}{l}\text { Anxiety/Depression } \\
\mathbf{n}(\%)\end{array}$} \\
\hline No problems & $142(71.7)$ & $182(91.9)$ & $87(43.9)$ & $45(22.7)$ & $129(65.2)$ \\
Any problems & $56(28.3)$ & $16(8.1)$ & $111(56.1)$ & $153(77.3)$ & $69(34.8)$ \\
\hline Total & $198(100)$ & $198(100)$ & $198(100)$ & $198(100)$ & $198(100)$ \\
\hline
\end{tabular}

EQ-5D-3L, EuroQoL-five-dimensional-three level.

the most significant barrier reported. Despite efforts by physiotherapy clinics to offer virtual visits, only $41.4 \%$ of patients were able to access this service, and of these patients only $42.9 \%$ reported that the visits were helpful. Postoperative rehabilitation is an essential component of a successful and timely recovery following restorative surgery. ${ }^{6}$ Without access to hands-on care, patients are at risk of complications including swelling, stiffness and weakness. ${ }^{78}$ These complications can contribute to a prolonged recovery and, in some cases, to additional surgical interventions. Accordingly, limited access to physiotherapy (particularly during the critical, early stages of recovery) can contribute to poor outcomes and increased healthcare costs.

With respect to virtual orthopaedic care, this study demonstrated that although $61.3 \%$ of postoperative patients found it to be beneficial, the majority stated that it did not replace an inperson visit. Although efforts have been made to improve virtual orthopaedic care by providing tips and guidelines to optimise the experience, ${ }^{9}$ many patients (and surgeons) believe that a hands-on assessment is essential to appropriately guide the course of recovery. In the setting of restorative orthopaedic surgery, virtual care may be viewed as an adjunct or alternative in select scenarios, but based on the information obtained in this survey, likely cannot replace inperson visits.

\section{CONCLUSION}

This study highlighted the significant impact of COVID-19related healthcare closures on preoperative and postoperative sport medicine orthopaedic surgery patients. The findings clearly demonstrated that restorative procedures play an important role in maintaining physical and emotional health, as well as returning individuals to work and leisure activities. The study also revealed that virtual care did not adequately replace the hands-on assessments of allied care and orthopaedic specialists. As the healthcare system begins to reopen, it will be important to ensure that patients awaiting restorative procedures are triaged appropriately in order to reduce both the individual and societal burden of disease.

Acknowledgements The authors would like to thank their colleagues as well as the staff of Banff Sport Medicine for their support of this research initiative.
Contributors MK was involved in the study design, data analysis and was the primary author of the manuscript. J-AF, LAH and SK were involved in the study design, data analysis and editing of the manuscript.

Funding The authors have not declared a specific grant for this research from any funding agency in the public, commercial or not-for-profit sectors.

Competing interests None declared.

Patient consent for publication Not required.

Ethics approval Formal research and ethics board approval was not required as this was a quality assurance study.

Provenance and peer review Not commissioned; externally peer reviewed.

Data availability statement Complete data are available via email or electronic link on request. Please contact the corresponding author for details.

Open access This is an open access article distributed in accordance with the Creative Commons Attribution Non Commercial (CC BY-NC 4.0) license, which permits others to distribute, remix, adapt, build upon this work non-commercially, and license their derivative works on different terms, provided the original work is properly cited, an indication of whether changes were made, and the use is noncommercial. See: http://creativecommons.org/licenses/by-nc/4.0/.

\section{ORCID iDs}

Laurie Anne Hiemstra http://orcid.org/0000-0002-7795-9565

Sarah Kerslake http://orcid.org/0000-0001-5940-5000

\section{REFERENCES}

1 Phillips MR, Chang Y, Zura RD, et al. Impact of COVID-19 on orthopaedic care: a call for nonoperative management. Ther Adv Musculoskelet Dis 2020;12:1759720X20934276.

2 Jain A, Jain P, Aggarwal S. SARS-CoV-2 impact on elective orthopaedic surgery: implications for Post-Pandemic recovery. J Bone Joint Surg Am 2020;102:e68.

3 Zeegen EN, Yates AJ, Jevsevar DS. After the COVID-19 pandemic: returning to Normalcy or returning to a new normal? J Arthroplasty 2020;35:S37-41.

4 Janssen MF, Szende A, Cabases J, et al. Population norms for the EQ-5D-3L: a cross-country analysis of population surveys for 20 countries. Eur J Health Econ 2019;20:205-16.

5 Endstrasser F, Braito M, Linser M, et al. The negative impact of the COVID-19 lockdown on pain and physical function in patients with end-stage hip or knee osteoarthritis. Knee Surg Sports Traumatol Arthrosc 2020. doi:10.1007/s00167-020-06104-3. [Epub ahead of print: 18 Jun 2020].

6 Jack K, McLean SM, Moffett JK, et al. Barriers to treatment adherence in physiotherapy outpatient clinics: a systematic review. Man Ther 2010;15:220-8.

7 Grant JA. Updating recommendations for rehabilitation after $A C L$ reconstruction: a review. Clin J Sport Med 2013;23:501-2.

8 Yabroudi MA, Irrgang JJ. Rehabilitation and return to play after anatomic anterior cruciate ligament reconstruction. Clin Sports Med 2013;32:165-75.

9 Tanaka MJ, Oh LS, Martin SD, et al. Telemedicine in the era of COVID-19: the virtual orthopaedic examination. J Bone Joint Surg Am 2020;102:e57. 